GLASNIK MATEMATIČKI

Vol. 40(60)(2005), $313-315$

\title{
THREE-DIMENSIONAL HYPERBOLIC GEOMETRY WITH PLANES AND PLANE PARALLELISM AS ONLY PRIMITIVE NOTIONS
}

\author{
Victor PAMBuccian \\ Arizona State University, USA
}

\begin{abstract}
We show that Euclidean Möbius planes can be axiomatized in terms of circles and circle-tangency, and that 3-dimensional hyperbolic geometry can be axiomatized in terms of planes and plane-parallelism.
\end{abstract}

Euclidean Möbius planes have been axiomatized in the 1950s, and a particularly simple axiom system, in terms of points, circles, incidence I, and orthogonality $\perp$ can be found in [1, III.2]. The axiom system, which will be referred to as $\Sigma$, consists of (M I), (M II), (M III) (p. 205), (M) (p. 218), as well as (O I), (O II), (O III) (p. 240), (O IV), (O V), (O VI) (pp. 245, 246, 248).

In 1905 H. Liebmann [5] pointed out, and H. S. M. Coxeter has tirelessly reminded us since the mid-sixties of its existence (in most detail in [2], [3]), the existence of an isomorphism between hyperbolic three-space and the inversive plane. Under this isomorphism planes in hyperbolic three-space correspond to circles in the inversive plane.

We have shown in [6] that circles and circle-orthogonality (or another binary notion related to the inversive distance between two circles) can serve as primitive notions for Euclidean Möbius planes, and that the corresponding notions can axiomatize 3-dimensional hyperbolic geometry.

2000 Mathematics Subject Classification. 51M10, 51B10, 03B30.

Key words and phrases. Hyperbolic space, Euclidean Möbius plane, axiom system.

This paper was written while the author was at the University of Bialystok with a Fulbright grant. I thank both the Polish-U.S. Fulbright Commission for the grant and the Institute of Mathematics of the University iof Białystok, and in particular Professor Krzysztof Prażmowski, for the hospitality. 
We will show in the present note that Benz's axiom system can be rephrased with circles as the only variables and circle tangency, to be denoted by $\tau$, as the only predicate, and thus that three-dimensional hyperbolic geometry over Euclidean fields can be axiomatized in terms of planes and parallelism.

To translate the axioms for Euclidean Möbius planes in a language with circles and tangency, we need to show how one can interpret 'points', as well as the relation of incidence, 'point $P$ is on circle $h$ ' and orthogonality, 'circle $a$ is orthogonal to circle $b$, in the language of $\tau$.

(i) A point is defined to be a pair of tangent circles $\left(h_{1}, h_{2}\right)$.

(ii) Point $P=\left(h_{1}, h_{2}\right)$ is leaning on sphere $h_{3}$, in symbols $\left(h_{1}, h_{2}\right) / h_{3}$, if $h_{3}$ is equal to $h_{1}$ or $h_{2}$, or else if $h_{3}$ is tangent to $h_{i}$ for $i \in\{1,2\}$, and if any two different circles $m_{1}$ and $m_{2}$ which are tangent to all the $h_{i}$ for $i=1,2,3$, are tangent to each other as well, i. e. with $\bmod 3$ addition in the indices,

$$
\begin{aligned}
\left(h_{1}, h_{2}\right) / h_{3}: \Leftrightarrow \quad & \left(\tau ( h _ { 1 } h _ { 2 } ) \wedge ( h _ { 3 } = h _ { 1 } \vee h _ { 3 } = h _ { 2 } ) \vee \left\{\bigwedge_{i=1}^{3} \tau\left(h_{i} h_{i+1}\right)\right.\right. \\
& \left.\wedge\left[\left(\forall m_{1} m_{2}\right)\left(m_{1} \neq m_{2} \bigwedge_{1 \leq i \leq 2,1 \leq j \leq 3} \tau\left(m_{i} h_{j}\right)\right) \rightarrow \tau\left(m_{1} m_{2}\right)\right]\right\} .
\end{aligned}
$$

(iii) Point $P=\left(h_{1}, h_{2}\right)$ is incident with (or on) circle $h_{3}$, in symbols $P$ I $h_{3}$, if $P$ is leaning on $h_{3}$ or else if $h_{3}$ is not tangent to any circle $h$ on which $P$ is leaning.

(iv) Two points $P=\left(h_{1}, h_{2}\right)$ and $Q=\left(l_{1}, l_{2}\right)$ are equal if and only if $P$ is on both $l_{1}$ and $l_{2}$.

(v) $k \perp k^{\prime}: \Leftrightarrow k \neq k^{\prime} \wedge(\exists A B C u v) A \neq B \wedge\left(A, B\right.$ I $\left.k, k^{\prime}\right) \wedge C$ I $k$ $\wedge \tau\left(u k^{\prime}\right) \wedge \tau\left(v k^{\prime}\right) \wedge \tau(u v) \wedge(A, C \mathrm{I} u) \wedge(B, C \mathrm{I} v)$.

Definitions (i)-(iv) are taken from [7], and (v) from [4]. In definition (v) and below $\left(A_{1}, \ldots, A_{n} \mathrm{I} h_{1}, \ldots, h_{m}\right)$ stands for $\bigwedge_{1 \leq i \leq n, 1 \leq j \leq m} A_{i} \mathrm{I} h_{j}$, and points and incidence are to be considered as abbreviations, in other words we consider the definiens to be a sentence expressed in terms of circles and $\tau$. We can now rephrase all the axioms in $\Sigma$ as axioms in the language with circles as the only variables and $\tau$ as the only predicate. Let $\Sigma_{0}$ denote the axiom system obtained in this manner. Let $\Sigma_{1}:=\Sigma_{0} \cup\{(1)\}$, where

$$
\tau(a b) \leftrightarrow(\exists P)\left(\forall P^{\prime}\right)(P \mathrm{I} a, b) \wedge\left(\left(P^{\prime} \mathrm{I} a, b\right) \rightarrow P=P^{\prime}\right),
$$

in which we consider again the point variables as having been replaced by two circle variables, as well as point-circle incidence and point equality having been replaced by their respective definitions, such that (1) is actually a sentence in terms of circles and $\tau$.

Given that in every model of $\Sigma_{0}$, if we interpret sets of two tangent circles as points, and define the incidence and the orthogonality as done in (i)-(v), 
points, circles, incidence, and orthogonality have the desired interpretations, the definiens in (1) says precisely that the circles $a$ and $b$ are tangent, so that in every model of $\Sigma_{1}$ circles and $\tau$ must have the desired interpretation. We have thus shown that

THEOREM 1. Euclidean Möbius planes are axiomatizable in terms of circles and circle tangency. Three-dimensional hyperbolic space is axiomatizable in terms of planes and parallelism.

Given that ascending chains of models of 3-dimensional hyperbolic geometry with planes and parallelism as primitive notions must be models of hyperbolic geometry, by the Eoś-Suszko-Chang theorem there must be a $\forall \exists$ axiom system for it (and for Euclidean Möbius planes as well). Given that the axiom system obtained by the rephrasing described above is however not such an axiom system, it would be of interest to find a $\forall \exists$-axiom system for these two theories.

\section{REFERENCES}

[1] W. Benz, Vorlesungen über Geometrie der Algebren, Springer-Verlag, Berlin 1973.

[2] H. S. M. Coxeter, The inversive plane and hyperbolic space, Abh. Math. Sem. Univ. Hamburg 29 (1966), 217-242 .

[3] H. S. M. Coxeter, Non-Euclidean geometry, Sixth edition, MAA Spectrum, Mathematical Association of America, Washington, DC, 1998.

[4] G. Ewald, Über den Begriff der Orthogonalität in der Kreisgeometrie, Math. Ann. 131 (1956), 463-469.

[5] H. Liebmann, Nichteuklidische Geometrie, B. G. Teubner, Leipzig, 1905.

[6] V. Pambuccian, Binary relations as single primitive notions for hyperbolic three-space and the inversive plane, Indag. Math. (N. S.) 11 (4) (2000), 587-592.

[7] V. Pambuccian, Sphere tangency as single primitive notion for hyperbolic and Euclidean geometry, Forum Math. 15 (2003), 943-947.

V. Pambuccian

Department of Integrative Studies

Arizona State University - West campus

P. O. Box 37100, Phoenix AZ 85069-7100

USA

E-mail: pamb@math.west.asu.edu

Received: 11.10.2004. 